\title{
Long non-coding RNA NEAT1 increases the aggressiveness of gastric cancer by regulating the microRNA-142-5p/JAG1 axis
}

\author{
YANMING ZHANG ${ }^{1}$, LIYING CHANG ${ }^{2}$, QUNMEI WU ${ }^{3}$ and FANG ZUO \\ ${ }^{1}$ Department of General Medicine, Yantaishan Hospital, Yantai, Shandong 264001; ${ }^{2}$ Health Management Center, \\ Qingdao Tumor Hospital; ${ }^{3}$ Health Management Center, Qingdao Central Hospital, Qingdao, Shandong 266042; \\ ${ }^{4}$ Department of Gastroenterology, Jinan Central Hospital, Jinan, Shandong 250010, P.R. China
}

Received October 15, 2019; Accepted February 25, 2021

DOI: $10.3892 /$ etm.2021.10294

\begin{abstract}
Gastric cancer has been indicated to have a high recurrence rate in China. Previous studies have revealed that long non-coding RNA nuclear-enriched abundant transcript 1 (NEAT1) exerted critical roles in cancers. Therefore, the present study aimed to determine the function of NEAT1 and explore the unknown molecular mechanisms of gastric cancer pathogenesis. Reverse transcription-quantitative PCR assay was used to examine the expression of NEAT1, microRNA (miR)-142-5p and jagged1 (JAG1) in gastric cancer. Cell Counting Kit-8 and Transwell assays were conducted to examine cell proliferation, migration and invasion. The protein expression levels of N-cadherin, Vimentin, E-cadherin and JAG1 were quantified by western blot assay. The associations among NEAT1, miR-142-5p and JAG1 were confirmed by dual-luciferase reporter assay and RNA immunoprecipitation. The effects of NEAT1 silencing on tumor growth were evaluated by tumor xenografts. The results indicated that NEAT1 was highly expressed in tumor tissues and cells compared with that in paracancerous tissues and the normal gastric epithelial cell line GES-1 and significantly associated with poor prognosis in gastric cancer. Functional analyses further demonstrated that NEAT1 knockdown suppressed proliferation, motility and tumor growth in vitro and in vivo. Mechanistically, NEAT1 sponged miR-142-5p to regulate JAG1 expression. In addition, the effects of NEAT1 knockdown on the proliferation, migration and invasion of gastric cancer cells could be rescued by miR-142-5p inhibitor, and JAG1 overexpression reversed the miR-142-5p-mediated effects on gastric cancer cells. These findings demonstrated that long non-coding RNA NEAT1 regulated gastric cancer progression by targeting the miR-142-5p/JAG1 axis.
\end{abstract}

Correspondence to: Mrs. Fang Zuo, Department of Gastroenterology, Jinan Central Hospital, 105 Jiefang Road, Jinan, Shandong 250010, P.R. China

E-mail: meigenggan2002rqb@126.com

Key words: long non-coding RNA, nuclear-enriched abundant transcript 1, microRNA-142-5p, jagged1, gastric cancer

\section{Introduction}

Gastric cancer is one of the most common malignancies, with $>1$ million estimated new cases and 784,000 deaths globally in 2018, which ranks the fifth among the most diagnosed malignancies worldwide and the third most common cause of cancer-associated mortality $(1,2)$. Excessive alcohol consumption, unbalanced diet and host genetic diversity are the main risk factors inducing gastric cancer (3). Finding the underlying molecular mechanisms is beneficial to diagnosis and therapy of gastric cancer, and it is urgent to identify molecular biomarkers associated with diagnostic and therapeutic targets of gastric cancer. A large amount of evidence has demonstrated that long non-coding RNAs (lncRNAs) exerted essential roles in biological processes, such as cell proliferation, migration, invasion, apoptosis and differentiation $(4,5)$. Previous studies have demonstrated that nuclear-enriched abundant transcript 1 (NEAT1) was upregulated in non-small cell lung cancer and esophageal squamous cell carcinoma tissues compared with that in their corresponding paracancerous tissues $(6,7)$. Moreover, NEAT1 has been indicated to function as a biomarker for the diagnosis and prognosis of colorectal cancer (8). Mechanistically, NEAT1 has been revealed to act as an oncogene and promote hepatocellular carcinoma progression via regulating heterogeneous nuclear ribonucleoprotein A2 (9). Conversely, knockdown of NEAT1 has been indicated to inhibit malignant glioma via targeting miR-449b-5p and lethal-7e $(10,11)$. NEAT1 has also been associated with poor prognosis and has been indicated to promote gastric cancer cell migration and invasion (12).

microRNAs (miRNAs) consist of 21-25 nucleotides and are endogenous non-coding RNAs that can affect the expression of mRNA by incompletely binding to the 3'-untranslated region (3'-UTR) of target genes (13). For example, Liu et al (14) reported that miR-375 enhanced cell proliferation capability by directly targeting p53 in gastric cancer. A previous study has indicated that downregulation of miR-124 was associated with breast cancer progression (15). In addition, miR-124 has been demonstrated to inhibit gastric cancer cell proliferation, but promote apoptosis via affecting enhancer of zeste homolog 2 and Rho-associated protein kinase $1(16,17)$. Similarly, Ma et al (18) indicated that miR-142-5p suppressed TGF- $\beta$-induced cancer cell growth and acted as a negative 
regulator of the TGF- $\beta$ pathway by targeting SMAD3. However, the mechanisms of miR-142 in inhibiting metastasis in gastric cancer are still undetermined.

Jagged1 (JAG1) is a vital regulator of the Notch signaling pathway, which participates in various cellular processes (19). JAG1 overexpression has been indicated to promote cell proliferation and inhibit apoptosis in multiple myeloma cells (20). In addition, JAG1 has been reported to be a target of miR-142 and facilitate the progression of gastric cancer via regulating the Notch signaling pathway $(15,21)$. In the current study, JAG1 was predicted as a target of miR-142-5p; however, the interaction between miR-142-5p and JAG1 in gastric cancer needs to be explored.

In the present study, NEAT1 expression was revealed to be upregulated in gastric cancer. Loss-of-function experiments were performed to verify the role NEAT1 in gastric cancer. Subsequently, the association between miR-142-5p, NEAT1 and JAG1 was identified by bioinformatics analysis, and it was revealed that NEAT1 exhibited a pro-carcinogenic role in gastric cancer progression by functioning as a sponge to target miR-142-5p and increase JAG1 expression.

\section{Materials and methods}

Clinical samples. A total of 33 pairs of biopsy samples used in the present study were obtained from patients with gastric cancer who underwent surgical resection at Yantaishan Hospital (Yantai, China) between April 2012 and December 2017. The clinicopathological features of these patients are presented in Table I. Inclusion criteria for patient recruitment: i) Patients with histologically confirmed first primary gastric cancer; ii) did not receive therapy before admission; and iii) willing to participate in follow up. Exclusion criteria for patients: i) Other clinical disorders (heart, liver, kidney or lung dysfunction; blood diseases, including leukemia, aplastic anemia, multiple myeloma, lymphoma, hemophilia and Mediterranean anemia; neurological illnesses, including seizures, major depression, schizophrenia, confused speech and Alzheimer disease) except gastric cancer; ii) received prior treatment; and iii) with history of previous malignancies. Written informed consent was obtained from all participants prior to surgery, and the protocols were approved by the Ethics Committee of Yantaishan Hospital (Yantai, China) and performed in accordance with the Declaration of Helsinki. Tumor samples and corresponding adjacent healthy tissues $>3 \mathrm{~cm}$ away from the primary tumors were promptly frozen in liquid nitrogen and stored at $-80^{\circ} \mathrm{C}$ until total RNA was extracted.

Cell culture and transfection. The human gastric cancer cell lines AGS and MKN-45 and the human normal gastric epithelial cell line GES-1 were purchased from China Center for Type Culture Collection. All cells were cultured in high glucose DMEM (Gibco; Thermo Fisher Scientific, Inc.) supplemented with 10\% FBS (Gibco; Thermo Fisher Scientific, Inc.) in a humidified atmosphere with $95 \%$ air $/ 5 \%$ $\mathrm{CO}_{2}$ at $37^{\circ} \mathrm{C}$.

Specific small interfering RNA (siRNA) against NEAT1 (si-NEAT1, sense, 5'-UGAUGGAGACGGAGCUGAAUU-3' and antisense, 5'-UUCAGCUCCGUCUCCAUCAUU-3') and its negative control (NC) si-NC (sense, 5'-UUCUCCGAACGU
GUCACGU-3' and antisense, 3'-ACGUGACACGUUCGG AGAA-5'), overexpression plasmid for NEAT1 and overexpression plasmid for JAG1 (JAG1) and their NC (pcDNA), miR-142-5p inhibitor (in-miR-142-5p, 5'-AGUAGUGCUUUC UACUUUAUG-3') and its NC (in-miR-NC, 5'-CGGUACGAU CGCGGCGGGAUAUC-3'), miR-142-5p mimic (miR-142-5p, 5'-CAUAAAGUAGAA AGCACUACU-3') and its NC (miR-NC, 5'-UCACAACCUCCUAGAAAGAGUAGA-3'), as well as specific short hairpin RNA (shRNA) against NEAT1 (sh-NEAT1, sense, 5'-CACCGGAAGGCAGGGAGAGGT AGA ACGAAT TCTACC TCTCCCTGCCTTCC-3' and antisense, 5'-AAAAGGAAGGCAGGGAGAGGTAGAATT CGTTCTACCTCTCCCTGCCTTCC-3') and its NC (sh-NC, sense, 5'-CACCGTTCTCCGAACGTGTCACGTCAAGAG ATTACGTGACACGTTCGGAGA ATT TTT TG-3' and antisense, 5'-GATCCAAAAAATTCTCCGAACGTGTCA CGTAATCTCTTGACGTGACACGTTCGGAGAAC-3') were purchased from Guangzhou RiboBio Co., Ltd. Lipofectamine $^{\circledR} 2000$ reagent (Thermo Fisher Scientific, Inc.) was used to transfect the aforementioned oligonucleotides or plasmids into AGS and MKN-45 cells at $37^{\circ} \mathrm{C}$. AGS and MKN-45 cells were collected at $48 \mathrm{~h}$ post-transfection for further analyses. The concentrations of miR-142-5p mimic and miR-142-5p inhibitor for transfection were $60 \mathrm{nM}$. The concentrations of overexpression plasmid for JAG1 and sh-NEAT1 for transfection were $1 \mu \mathrm{g}$.

$R N A$ isolation and reverse transcription quantitative $P C R$ $(R T-q P C R)$. Total RNA was extracted from gastric cancer tissue samples or AGS and MKN-45 cells using TRIzol ${ }^{\circledR}$ reagent (Thermo Fisher Scientific, Inc.) according to the manufacturer's instructions. cDNA for lncRNA/mRNA and miRNA was synthesized using PrimeScript ${ }^{\mathrm{TM}}$ RT Reagent kit (Takara Biotechnology Co., Ltd.) and TaqMan ${ }^{\mathrm{TM}}$ microRNA Reverse Transcription Kit (Thermo Fisher Scientific, Inc.) at $37^{\circ} \mathrm{C}$ for $15 \mathrm{~min}$, followed by $85^{\circ} \mathrm{C}$ for $15 \mathrm{sec}$. qPCR analysis was performed using QuantiTect SYBR-Green RT-PCR Kit (Qiagen, Inc.) on ABI 7500 HT system (Applied Biosystems; Thermo Fisher Scientific, Inc.) by following the thermocycling conditions: 1 cycle of $95^{\circ} \mathrm{C}$ for 3 min and 40 cycles of $95^{\circ} \mathrm{C}$ for $15 \mathrm{sec}, 60^{\circ} \mathrm{C}$ for $1 \mathrm{~min}$ and $72^{\circ} \mathrm{C}$ for $30 \mathrm{sec}$ followed by 1 cycle of extension at $72^{\circ} \mathrm{C}$ for $7 \mathrm{~min}$, with GAPDH (for lncRNA and mRNA) or endogenous small nuclear RNA U6 (for miRNA) as the internal control. Relative expression was determined using the $2^{-\Delta \Delta \mathrm{Cq}}$ method (22). The primers used are listed as follows: miR-142-5p (forward, 5'-GCCGAGCATAAAGTAGAAAG-3'; reverse, 5'-CTCAACTGGTGTCGTGGA-3'); JAG1 (forward, 5'-GGGGCAACACCTTCAACCTC-3'; reverse, 5'-CCACGC CTCCACAAGCAAC-3'); NEAT1 (forward, 5'-AATTCTGTT ACGTCATGT-3'; reverse, 5'-TTTCTAATGAGTTTAGAA CTCAAAC-3'); GAPDH (forward, 5'-TCCCATCACCATCTT CCAGG-3'; reverse, 5'-GATGACCCTTTTGGCTCCC-3'); and U6 (forward, 5'-CTCGCTTCGGCAGCACA-3'; reverse, 5'-AACGCTTCACGAATTTGCGT-3').

Cell Counting Kit-8 (CCK-8) assay. AGS and MKN-45 cells were seeded into 96-well plates (Corning, Inc.) at a density of $5.0 \times 10^{3}$ cells per well. A total of $20 \mu \mathrm{l}$ of CCK- 8 solution were added into the wells and the cells were incubated for $4 \mathrm{~h}$ at $37^{\circ} \mathrm{C}$. Subsequently, cell viability was determined by 
Table I. Clinicopathological features of patients with gastric cancer $(n=33)$.

\begin{tabular}{|c|c|c|}
\hline Characteristics & $\mathrm{N}$ & $\%$ \\
\hline \multicolumn{3}{|l|}{ Sex } \\
\hline Female & 15 & 45 \\
\hline Male & 18 & 55 \\
\hline \multicolumn{3}{|l|}{ Age (years) } \\
\hline$<50$ & 16 & 48 \\
\hline$\geq 50$ & 17 & 52 \\
\hline \multicolumn{3}{|l|}{ Surgical technique } \\
\hline Total gastrectomy & 25 & 76 \\
\hline Partial gastrectomy & 8 & 24 \\
\hline \multicolumn{3}{|l|}{ Neoadjuvant therapy } \\
\hline No & 15 & 45 \\
\hline Yes & 18 & 55 \\
\hline \multicolumn{3}{|c|}{ Resection classification } \\
\hline R0 & 27 & 82 \\
\hline $\mathrm{R} 1$ & 6 & 18 \\
\hline \multicolumn{3}{|c|}{ Histopathological response } \\
\hline Minor & 18 & 55 \\
\hline Major & 15 & 45 \\
\hline \multicolumn{3}{|l|}{ Clinical T-stage } \\
\hline $\mathrm{T}_{1}$ & 2 & 6 \\
\hline $\mathrm{T}_{2}$ & 5 & 15 \\
\hline $\mathrm{T}_{3}$ & 22 & 67 \\
\hline $\mathrm{T}_{4}$ & 4 & 12 \\
\hline \multicolumn{3}{|l|}{ Clinical N-stage } \\
\hline $\mathrm{N}_{0}$ & 1 & 3 \\
\hline $\mathrm{N}_{1}$ & 6 & 18 \\
\hline $\mathrm{N}_{2}$ & 21 & 64 \\
\hline $\mathrm{N}_{3}$ & 5 & 15 \\
\hline
\end{tabular}

measuring the absorbance at $450 \mathrm{~nm}$ on a microplate reader (Applied Biosystems; Thermo Fisher Scientific, Inc.).

Cell migration and invasion assay. Transwell chambers $(8-\mu \mathrm{m}$ pore size; EMD Millipore) were used to investigate the migration and invasion of AGS and MKN-45 cells. For the detection of migration, cells $\left(2 \times 10^{5}\right.$ cells in serum-free DMEM) were placed into the upper chamber, while the lower chamber contained high glucose DMEM with $10 \%$ FBS for the induction of migration. After incubation at $37^{\circ} \mathrm{C}$ for $48 \mathrm{~h}$, the cells that passed through the membrane were fixed with $20 \%$ methanol for $15 \mathrm{~min}$ at room temperature and stained with $0.1 \%$ crystal violet for $30 \mathrm{~min}$ at room temperature. The migrated cells were counted under a light microscope (Guangzhou Micro-shot Technology Co., Ltd.) at x100 magnification. In addition, $12.5 \mathrm{mg}$ Matrigel (BD Biosciences) in $50 \mathrm{ml}$ PBS was added to the upper chamber and then incubated at $37^{\circ} \mathrm{C}$ for $12 \mathrm{~h}$ for the invasion assay, the other steps being the same as aforementioned.

Dual-luciferase reporter assay. The online software LncBase v2 (http://diana.imis.athena-innovation.
gr/DianaTools/index.php?r=lncBase/index) and microT-CDS v5.0 (http://diana.imis.athena-innovation.gr/DianaTools/index. php? $r=$ microT_CDS/index) were employed to predict potential targets of NEAT1 and miR-142-5p, respectively. The fragment of lncRNA NEAT1 containing the miR-142-5p binding sites [from nucleotide (nt) 803 to 823] was amplified using cDNA samples from AGS cells as a template with the following primers: Forward, 5'-CGGGCTTACCAGATGACC AG-3' and reverse, 5'-CGGGCTTACCAGATGACCAG-3'. PCR was performed using the High-Fidelity PCR Master Mix kit (Guangzhou RiboBio Co., Ltd) with the following thermocycling conditions: $3 \mathrm{~min}$ initial denaturation at $94^{\circ} \mathrm{C}$, 35 cycles of denaturation at $94^{\circ} \mathrm{C}$ for $10 \mathrm{sec}$, annealing at $60^{\circ} \mathrm{C}$ for $10 \mathrm{sec}$ and $72^{\circ} \mathrm{C}$ extension for $45 \mathrm{sec}$ and a final extension step at $72^{\circ} \mathrm{C}$ for $1 \mathrm{~min}$. The fragment of JAG1 3'-UTR (from nt 4127 to 5940 ) containing the miR-142-5p binding sites (from nt 4502 to 4522) was amplified using cDNA samples from AGS cells as a template using the following primers: Forward, 5'-CAGCCTCTGAGGACAACACC-3' and reverse, 5'-GAC ATCAAAGTCTCCCCTCCC-3'. The sequences were cloned into pGL3 luciferase vector (Promega Corporation) to produce WT reporters (NEAT1 WT and JAG1 3'-UTR WT). In addition, NEAT1 mutant type (MUT) fragments and JAG1 3'-UTR MUT fragments carrying the point mutation of miR-142-5p binding sites were utilized to produce MUT reporters (NEAT1 MUT and JAG1 3'-UTR MUT) in the same way. Sequence synthesis, mutations and the construction of the luciferase reporter vector were conducted by Guangzhou RiboBio Co., Ltd.

The reporter vectors $(1 \mu \mathrm{g} / \mathrm{ml}$ final concentration) and miR-142-5p (20 nM final concentration) or miR-NC (20 nM final concentration) were co-transfected into AGS and MKN-45 cells using Lipofectamine 2000 (Thermo Fisher Scientific, Inc.), and $36 \mathrm{~h}$ following transfection cells were lysed and luciferase activity was detected using Dual-Luciferase Reporter Assay System (Promega Corporation), with Renilla luciferase activity as the transfection control.

RNA immunoprecipitation (RIP) assay. RIP assays were performed at $24 \mathrm{~h}$ post-transfection using the EZ-Magna RIP $^{\text {тм }}$ RNA Binding Protein Immunoprecipitation kit (EMD Millipore) according to the manufacturer's protocols. In brief, AGS and MKN-45 cells were lysed using RIP buffer. Prior to incubating with equal amounts of cell lysates (200-400 $\mu \mathrm{g} / 200 \mu \mathrm{l}$ per $\mathrm{RIP}$ reaction) overnight at $4^{\circ} \mathrm{C}$, $50 \mu \mathrm{l}(500 \mu \mathrm{g})$ protein $\mathrm{A} / \mathrm{G}$ magnetic beads $(10 \mathrm{mg} / \mathrm{ml})$ were coupled with antibodies against $\operatorname{IgG}(5 \mu \mathrm{g}$ per RIP reaction; cat. no. PP64B; EMD Millipore) or argonaute2 (Ago2; 1:50, cat. no. 2897; Cell Signaling Technology, Inc.) for $30 \mathrm{~min}$ at room temperature. The immunoprecipitate pellet was then collected using the magnetic separator by centrifugation $(1,000 \mathrm{x} \mathrm{g}, 1 \mathrm{~min})$ at room temperature, before incubation with proteinase $\mathrm{K}$ at $55^{\circ} \mathrm{C}$ for $30 \mathrm{~min}$ to remove the proteins. The RNA was purified using phenol: Chloroform: Isoamyl alcohol and centrifuged at $12,000 \mathrm{x}$ g for $10 \mathrm{~min}$ at room temperature. RNA in the aqueous phase was collected and then precipitated with absolute ethanol at $-80^{\circ} \mathrm{C}$ overnight and then analyzed by RT-qPCR.

Western blot assay. AGS and MKN-45 cells and tumor tissue samples were lysed using RIPA buffer(ThermoFisher Scientific, 
A

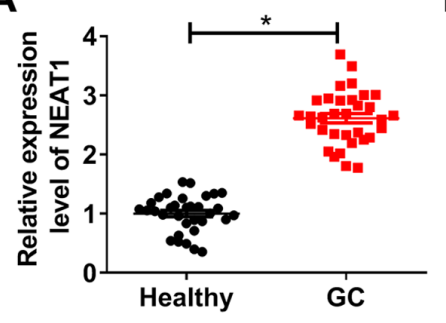

B

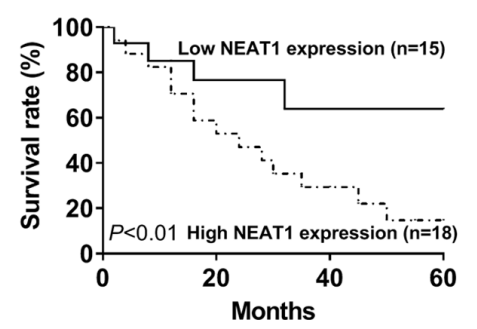

C

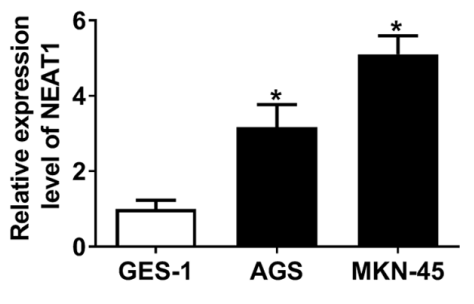

Figure 1. Expression level of NEAT1 in gastric cancer tissues and cells. (A) Expression level of NEAT1 in gastric cancer tissues and neighboring healthy tissues examined by RT-qPCR ( $\mathrm{n}=33$ pairs). (B) Survival curves of patients with gastric cancer (Kaplan-Meier curve with log-rank test). (C) Expression level of NEAT1 in two gastric cancer cell lines (AGS and MKN-45) compared with the human normal gastric epithelial cell line GES-1 determined by RT-qPCR. ${ }^{*} \mathrm{P}<0.05$. NEAT1, nuclear-enriched abundant transcript 1; RT-qPCR, reverse transcription-quantitative PCR.

Inc.) with protease inhibitors (Thermo Fisher Scientific, Inc.). Subsequently, protein concentration was measured using BCA Protein Assay Kit (Thermo Fisher Scientific, Inc.). A total of $50 \mu \mathrm{g}$ protein/sample were separated via $12 \%$ SDS-PAGE and transferred onto PVDF membranes (Millipore). Subsequently, membranes were blocked with $5 \%$ nonfat milk and incubated with primary antibodies at $4^{\circ} \mathrm{C}$ overnight, and anti- $\beta$-actin (cat. no. sc-8432; 1:2,000 dilution; Santa Cruz Biotechnology, Inc.) served as a reference. Subsequently, the membranes were incubated with HRP-conjugated secondary antibodies (cat. no. sc-2005; 1:2,000 dilution; Santa Cruz Biotechnology, Inc.). Finally, signal intensity was visualized with enhanced chemiluminescence detection kit (Thermo Fisher Scientific, Inc.). In addition, Image Pro Plus software v.6.0 (Media Cybernetics, Inc.) was used to determine the protein relative expression level. The primary antibodies were as follows: Anti-N-cadherin (cat. no. sc-8424; 1:1,000 dilution), anti-Vimentin (cat. no. sc-6260; 1:1,000 dilution), anti-E-cadherin (cat. no. sc-8426; 1:1,000 dilution) and anti-JAG1 (cat. no. sc-390177; 1:1,000 dilution; all from Santa Cruz Biotechnology, Inc.).

In vivo experiment. All animal experiments were performed according to the guide for the care and use of laboratory animals of the Ministry of Science and Technology of China (23). In total, 12 4-week-old male BALB/c nude mice (weight, $18 \pm 2 \mathrm{~g}$ ) were purchased from The Shanghai Experimental Animal Center and kept at a temperature $22 \pm 1^{\circ} \mathrm{C}$ and humidity $55 \pm 5 \%$, 12-h light/dark cycle, with free access to clean food and water. The stable AGS cell line expressing sh-NEAT1 or sh-NC was established by transfecting AGS cells with sh-NEAT1 or sh-NC and followed by selection with $4 \mu \mathrm{g} / \mathrm{ml}$ puromycin (Sigma-Aldrich; Merck KGaA) for 4-5 weeks. Stable cell lines were maintained in the high glucose DMEM medium with $10 \% \mathrm{FBS}$ and $1 \mu \mathrm{g} / \mathrm{ml}$ puromycin. AGS cells $\left(5 \times 10^{6} / 100 \mu \mathrm{l}\right.$ $\mathrm{PBS} /$ mice) stably transfected with sh-NEAT1 or sh-NC were subcutaneously injected into the mice at right flank of nude mice. Tumor growth was monitored with calipers by measuring the maximum (a) and minimum (b) length of the tumor every 7 days. Tumor volume (V) was assessed as follows: $\mathrm{V}=1 / 2 \mathrm{x} \mathrm{ab}^{2}$. All mice were sacrificed by flowing $\mathrm{CO}_{2}$ inhalation $(45 \%$ of the cage vol/min; the heartbeat and pupils of the mice were examined to ensure death) on day 35 after inoculation, and tumors were weighted and used for subsequent experiments. The Institutional Animal Care and Use Committee of
Yantaishan Hospital (Yantai, China) authorized all animal experiments.

Statistical analysis. All data are presented as the mean \pm standard deviation from $\geq$ three independent experiments. The difference between two groups was examined by Student's t-test (paired Student's t-test was used to examine statistically significant differences between tumor and adjacent non-tumor tissues of the same individual, while unpaired Student's t-test was used for two unpaired groups), and differences among $\geq 3$ groups were analyzed using one-way ANOVA followed by Bonferroni's post hoc test using GraphPad Prism 7 (GraphPad Inc.). P<0.05 was considered to indicate a statistically significant difference. Survival curves of patients with gastric cancer was performed by Kaplan-Meier curve analysis with log-rank test. Pearson's correlation analysis was used to determine correlation relationship.

\section{Results}

NEAT1 is highly expressed in gastric cancer tissues and cells. The expression of NEAT1 in gastric cancer tissues and cells was firstly examined by RT-qPCR. As presented in Fig. 1A, NEAT1 expression level was significantly upregulated in 33 cases of gastric cancer compared with matched healthy tissues. The data indicated that low expression of NEAT1 (according to the median of NEAT1 level in tumor tissues) was associated with high survival rate, while patients with high level of NEAT1 presented low survival rate (Fig. 1B). In addition, the expression level of NEAT1 in human gastric cancer cell lines (AGS and MKN-45 cells) was increased compared with the human normal gastric epithelial cell line GES-1 (Fig. 1C). Therefore, the results indicated that high expression of NEAT1 was associated with a poor prognosis of gastric cancer.

NEAT1 knockdown suppresses the proliferation, migration and invasion of gastric cancer cells. To understand the effect of NEAT1 on gastric cancer progression, a loss-of-function experiment was conducted. The level of NEAT1 expression was notably decreased in AGS and MKN-45 cells transfected si-NEAT1 (Fig. 2A and B). To explore the proliferative ability of AGS and MKN-45 cells transfected with si-NEAT1 or si-NC, CCK-8 assay was performed, which indicated that NEAT1 knockdown inhibited the proliferation of gastric cancer cells 

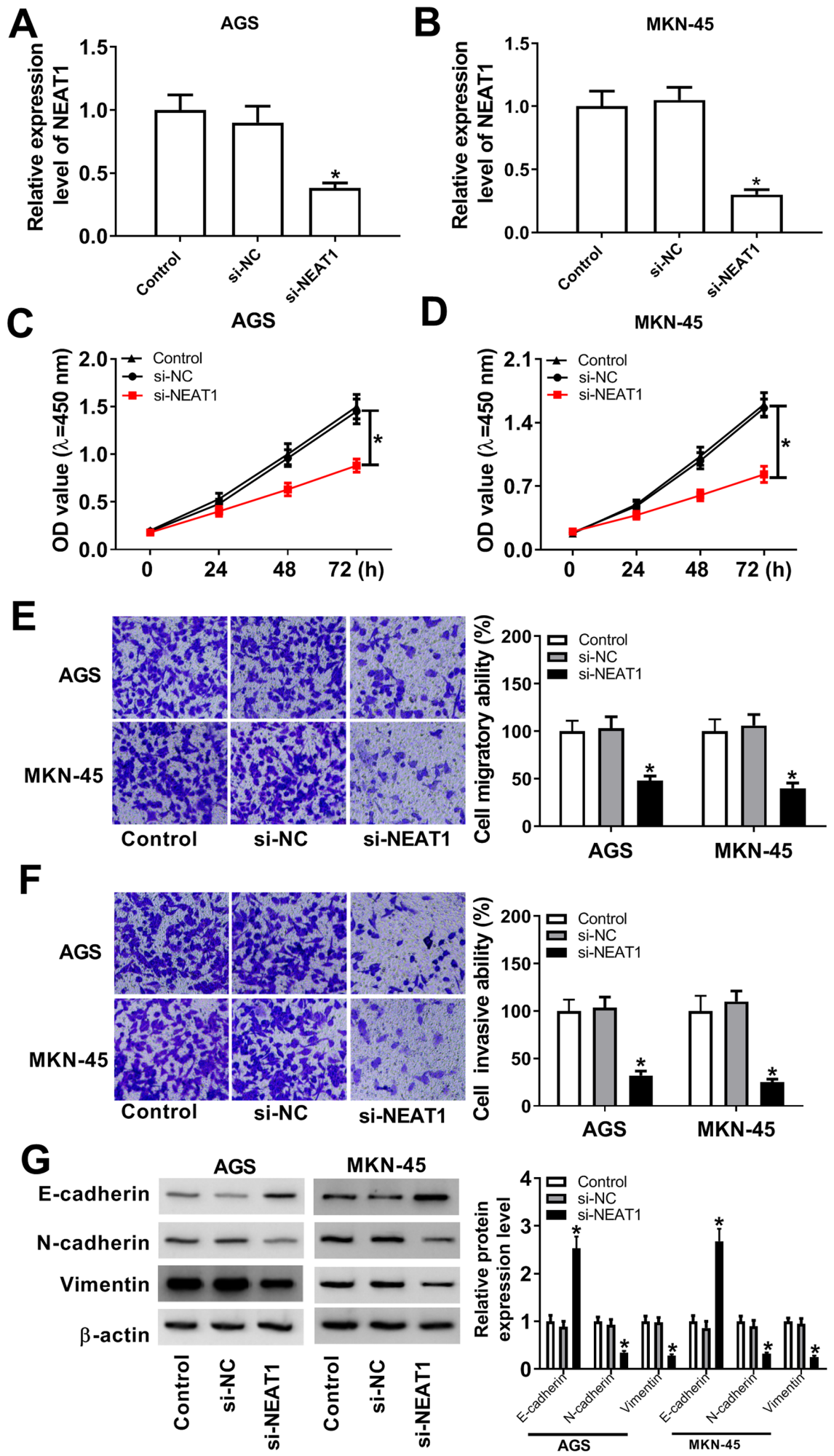

Figure 2. Effect of NEAT1 knockdown on proliferation, migration and invasion in gastric cancer cells. AGS and MKN-45 cells were transfected with si-NC or si-NEAT1. (A and B) Relative expression level of NEAT1 in AGS and MKN-45 cells determined by reverse transcription-quantitative PCR. (C and D) Cell viability of AGS and MKN-45 cells at the indicated time points analyzed with Cell Counting Kit-8 assay. (E and F) Cell migratory and invasive capacity of AGS and MKN-45 cells examined by Transwell assay. (G) Protein expression levels of E-cadherin, $\mathrm{N}$-cadherin and Vimentin determined by western blot assay. ${ }^{*} \mathrm{P}<0.05$. NEAT1, nuclear-enriched abundant transcript 1; si, small interfering; NC, negative control; OD, optical density.

(Fig. 2C and D). Gastric cancer cells transfected with si-NEAT1 exhibited a notable reduction in the number of migrated cells compared with the si-NC group (Fig. 2E). Consistent with the migration assay results, NEAT1 silencing suppressed the invasion of gastric cancer cells (Fig. 2F). Furthermore, N-cadherin and
Vimentin expression levels were decreased, but E-cadherin level was increased in the si-NEAT1 group compared with the si-NC group (Fig. 2G). Collectively, the results indicated that NEAT1 may serve an oncogenic role by enhancing proliferation, migration and invasion in gastric cancer cells. 
A

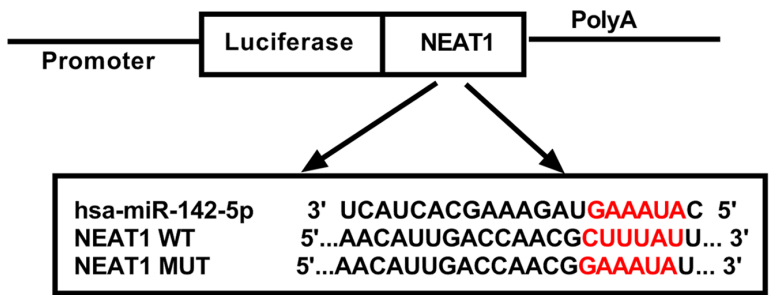

B

D
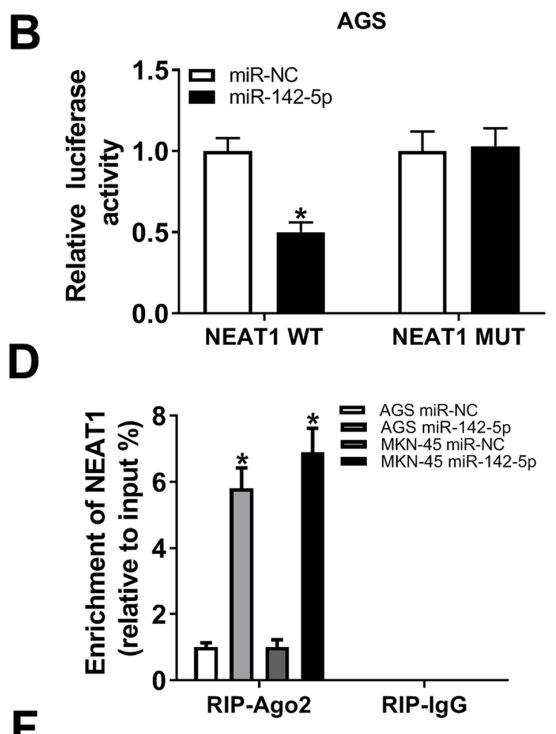

F
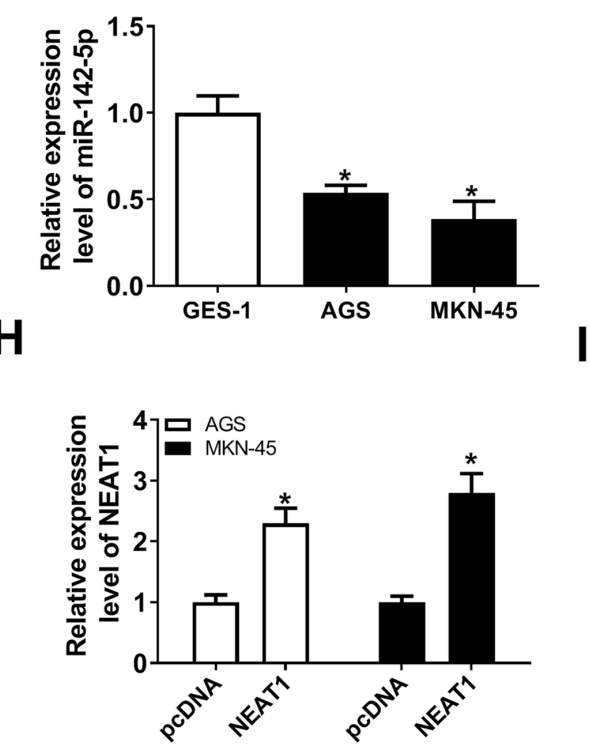

C

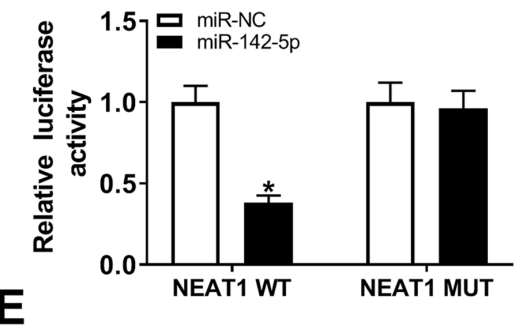

G
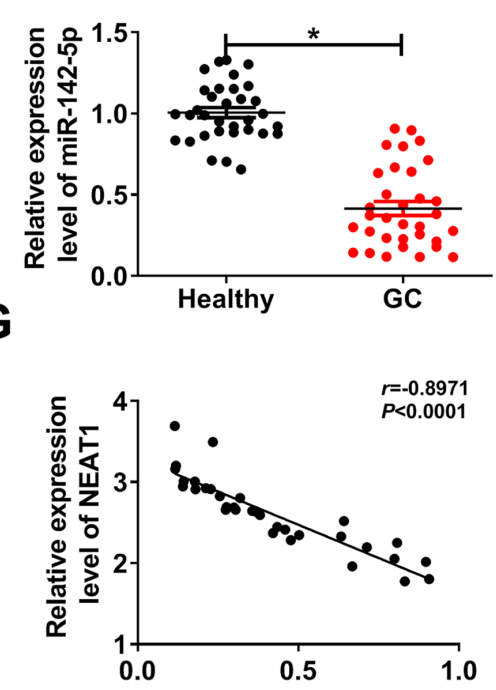

Relative expression level of miR-142-5p

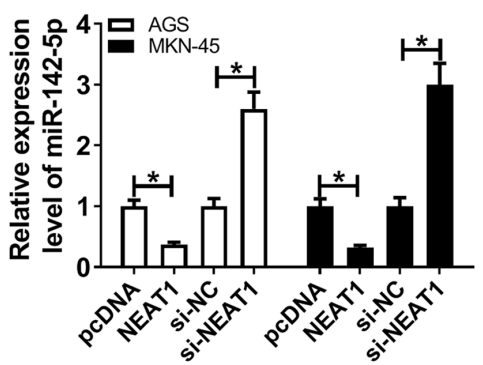

Figure 3. NEAT1 directly interacts with miR-142-5p. (A) Putative binding sites between NEAT1 and miR-142-5p and NEAT1 mutant sites. (B and C) Luciferase activity in AGS and MKN-45 cells co-transfected with NEAT1-WT or NEAT1-MUT luciferase reporter and miR-142-5p or miR-NC, respectively. (D) RIP assay in AGS and MKN-45 cells. (E and F) Expression level of miR-142-5p in gastric cancer tissues and cells determined by RT-qPCR. (G) Correlation analysis of NEAT1 and miR-142-5p expression levels. (H) Expression level of NEAT1 in AGS and MKN-45 cells transfected with pcDNA or NEAT1 overexpression vector. (I) Expression level of miR-142-5p in AGS and MKN-45 cells transfected with pcDNA, NEAT1, si-NC or si-NEAT1 determined by RT-qPCR. "P<0.05. NEAT1, nuclear-enriched abundant transcript 1; miR, microRNA; si, small interfering; NC, negative control; RT-qPCR, reverse transcription-quantitative PCR; RIP, RNA immunoprecipitation; WT, wild-type; MUT, mutant; Ago2, argonaute2.

NEAT1 directly interacts with miR-142-5p. Following bioinformatics analysis using LncBase v2, several miRNAs were predicted to serve as potential targets of NEAT1, including miR-761, miR-101-3p, miR-9-5p, miR-181b-5p, miR-194-5p and miR-142-5p. The expression level of these miRNAs in healthy tissues and gastric cancer tissues was assessed, and miR-142-5p was the most significantly decreased in gastric cancer tissues compared with healthy tissues (Fig. S1). miR-142-5p was therefore selected for subsequent analysis. The putative binding sites between NEAT1 and miR-142-5p are presented in Fig. 3A. The dual-luciferase reporter assay results indicated that miR-142-5p overexpression effectively suppressed the luciferase activity of the NEAT1-WT reporter in AGS and MKN-45 cells (Fig. 3B), but the luciferase 
A

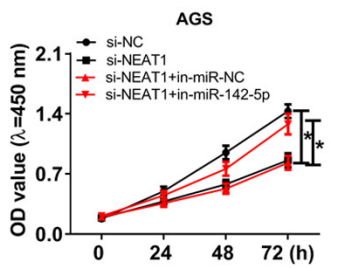

B

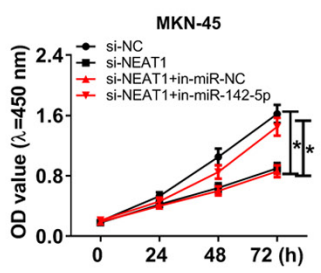

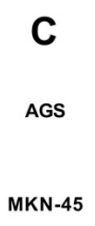
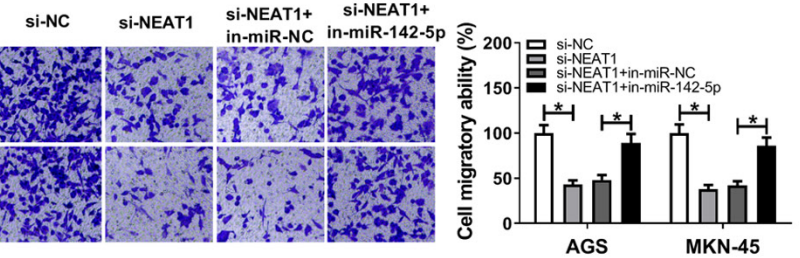
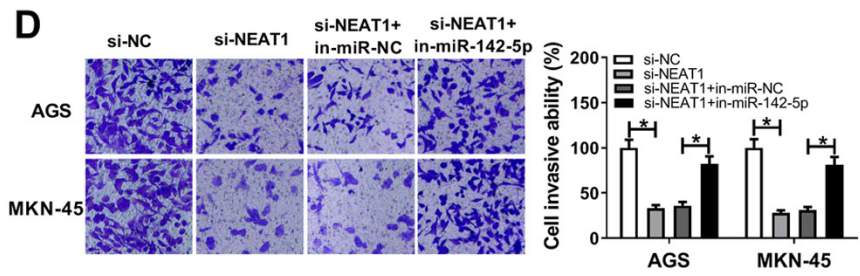

E
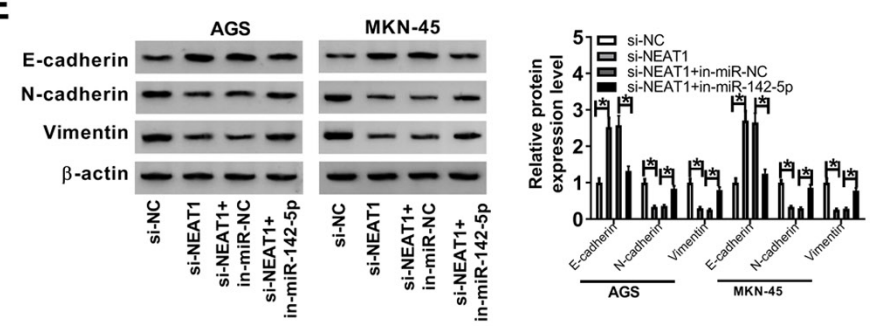

Figure 4. Effect of miR-142-5p inhibitor on the NEAT1 knockdown-mediated effects on proliferation, migration and invasion in gastric cancer cells. AGS and MKN-45 cells were transfected with si-NC, si-NEAT1, si-NEAT1 + in-miR-NC or si-NEAT1 + in-miR-142-5p. (A and B) Cell viability of transfected AGS and MKN-45 cells at the indicated time points analyzed with Cell Counting Kit-8 assay. (C and D) Migratory and invasive abilities of AGS and MKN-45 cells examined by Transwell assay. (E) Protein expression level of E-cadherin, N-cadherin and Vimentin in AGS and MKN-45 cells post-transfection determined by western blot assay. "P<0.05. NEAT1, nuclear-enriched abundant transcript 1; miR, microRNA; in, inhibitor; si, small interfering; NC, negative control; OD, optical density.

activity of the NEAT1-MUT reporter in gastric cancer cells exhibited no significant change compared with the control group (Fig. 3C). To further clarify the association between NEAT1 and miR-142-5p, RIP assay was performed to assess whether NEAT1 could interact with miR-142-5p. miR-142-5p overexpression resulted in a significant enrichment of NEAT1 in the Ago2 immunoprecipitation complex compared with the miR-NC control group (Fig. 3D). To investigate the regulatory association between NEAT1 and miR-142-5p, the relative expression level of miR-142-5p was firstly evaluated. miR-142-5p was indicated to be decreased in gastric cancer tumor tissues and cells compared with that in normal tissues and GES-1 cells (Fig. 3E and F). A negative correlation between NEAT1 and miR-142-5p expression level in gastric cancer tissues was also observed (Fig. 3G). Subsequently, RT-qPCR assay confirmed that NEAT1 expression level was notably increased in AGS and MKN-45 cells transfected with NEAT1 overexpression plasmid compared with control cells (Fig. 3H). miR-142-5p expression level was significantly decreased in cells transfected with NEAT1 overexpression vector, while miR-142-5p level was elevated in the NEAT1 knockdown group (Fig. 3I). All these data indicated that NEAT1 directly targeted miR-142-5p.

miR-142-5p inhibitor rescues the NEAT1 silencing-mediated effects on proliferation, migration and invasion in gastric cancer cells. The effects of NEAT1 knockdown along with miR-142-5p silencing on proliferation, migration and invasion in gastric cancer cells were further explored. miR-142-5p expression level was decreased in AGS and MKN-45 cells after transfection with miR-142-5p inhibitor, while it was increased in miR-142-5p mimic-transfected cells compared with control cells (Fig. S2A). Silencing of miR-142-5p reversed the reduction in cell viability in AGS and MKN-45 cells caused by NEAT1 knockdown (Fig. 4A and B). Similarly, Transwell assay indicated that miR-142-5p inhibitor attenuated the NEAT1 knockdown-mediated inhibitory effects on the migration and invasion of AGS and MKN-45 cells (Fig. 4C and D). Moreover, NEAT1 knockdown markedly decreased $\mathrm{N}$-cadherin and Vimentin expression levels, whereas E-cadherin was increased; however, co-transfection of si-NEAT1 and miR-142-5p inhibitor reversed these effects in AGS and MKN-45 cells (Fig. 4E). Taken together, these results indicated that NEAT1 directly targeted miR-142-5p to enhance the proliferative, migratory and invasive capability of gastric cancer cells.

JAG1 is a target of miR-142-5p in gastric cancer. The binding region of miR-142-5p on the 3'-UTR of JAG1 and its mutant are presented in Fig. 5A. As indicated in Fig. 5B and C, miR-142-5p overexpression decreased the luciferase activity of JAG1 3'-UTR WT rather than that of JAG1 3'-UTR MUT in AGS and MKN-45 cells. To further investigate whether JAG1 was involved in the development of gastric cancer, the expression of JAG1 in gastric cancer tissues and cells was examined by RT-qPCR. As presented in Fig. 5D and E, JAG1 expression level was significantly increased in gastric cancer tissues and cells compared with the corresponding controls. The negative correlation between miR-142-5p and JAG1 expression level is illustrated in Fig. 5F. A gain-of-function experiment revealed that the protein level of JAG1 was reduced in AGS and MKN-45 cells transfected with miR-142-5p mimic compared with miR-NC (Fig. 5G). Thus, it was concluded that miR-142-5p directly suppressed JAG1 expression in gastric cancer.

JAG1 overexpression abolishes the effects of miR-142-5p on proliferation, migration and invasion in gastric cancer cells. According to the aforementioned results, the overexpression of miR-142-5p inhibited JAG1 expression in gastric cancer cells. The expression level of JAG1 was increased in AGS and 
A

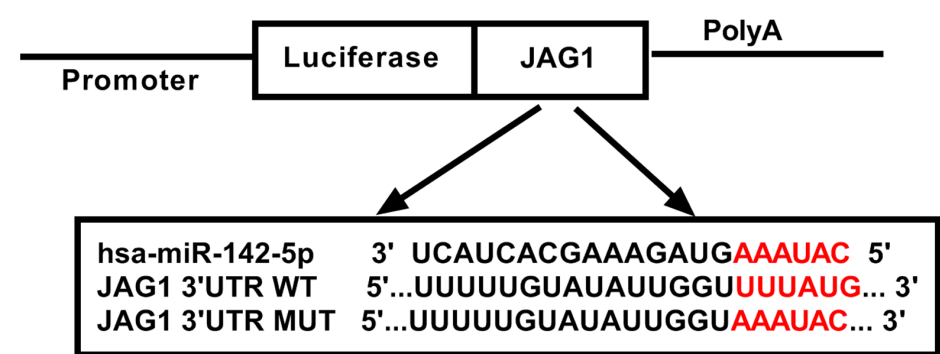

B

AGS
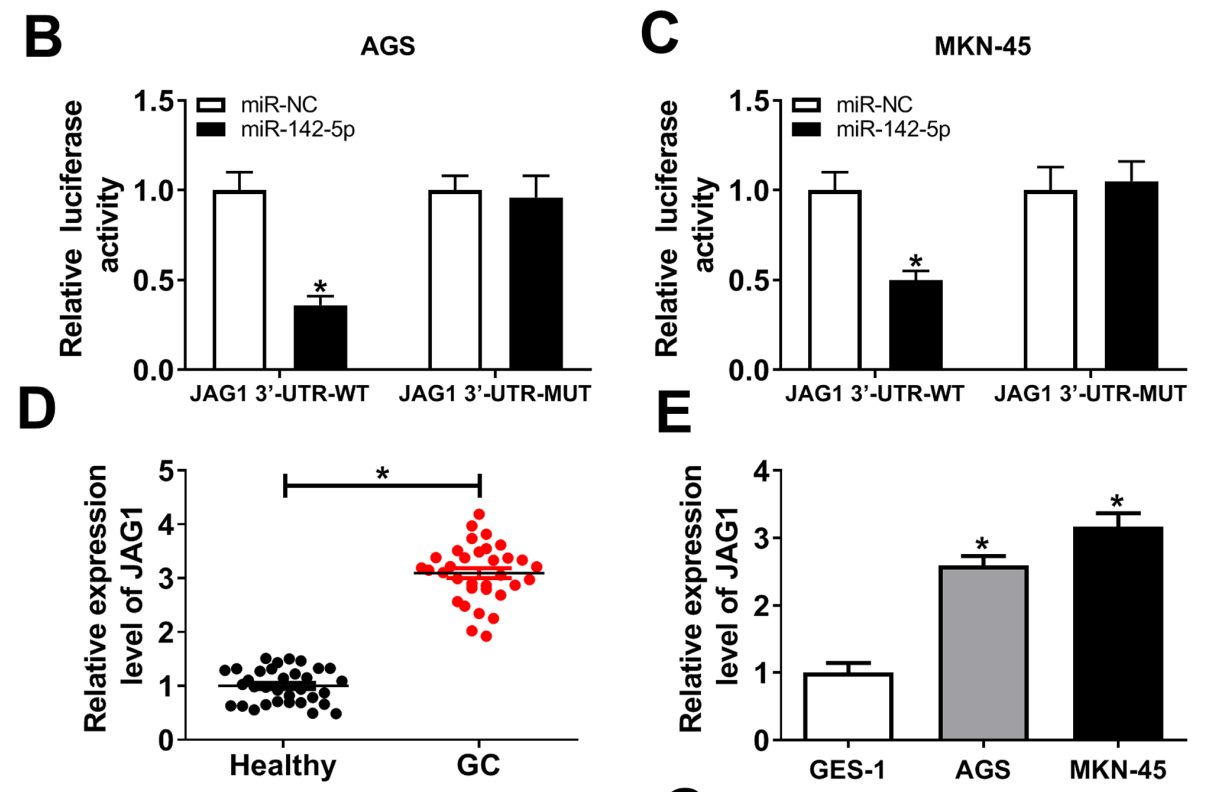

$\mathbf{F}$
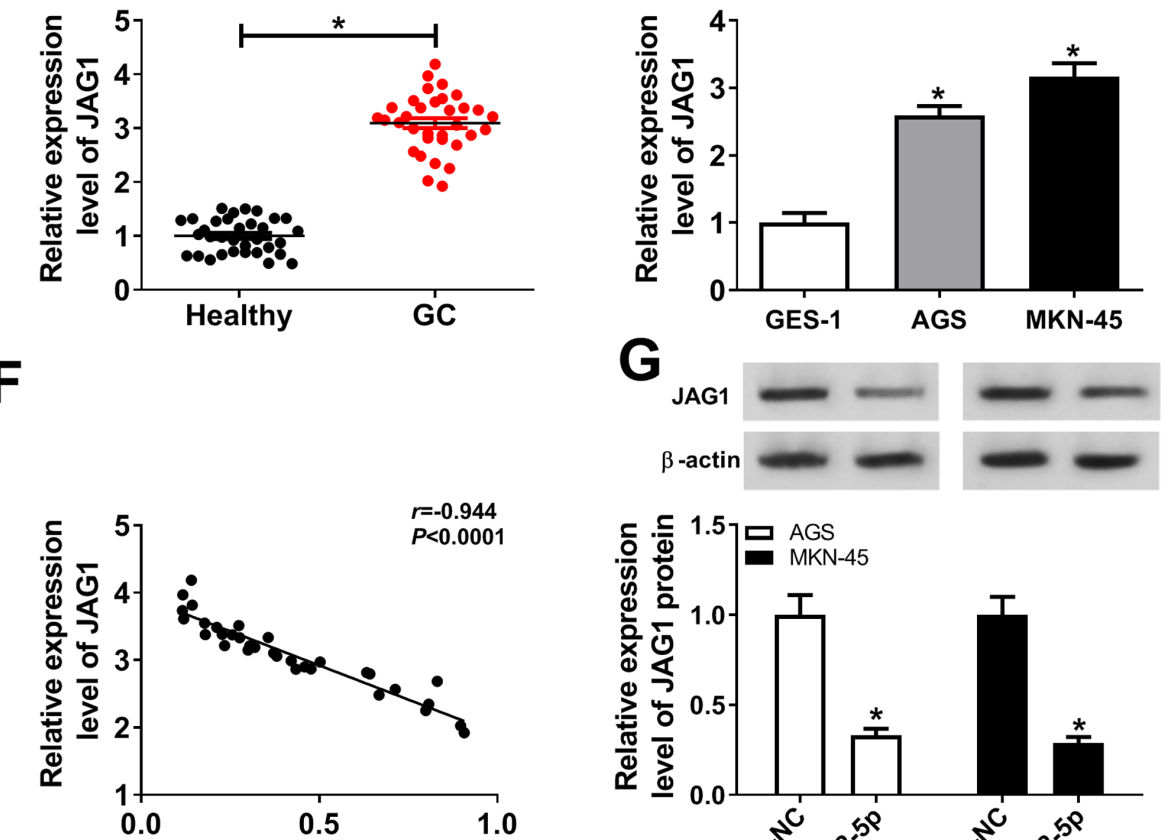

Relative expression level of miR-142-5p

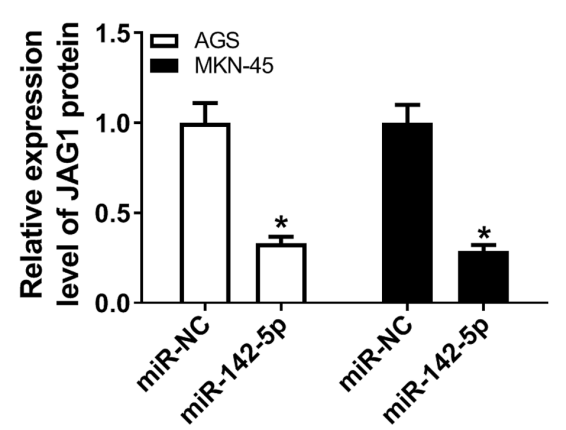

Figure 5. Direct inhibition of JAG1 expression by miR-142-5p in gastric cancer cells. (A) Predicted binding sequences of miR-142-5p on the 3'-UTR of JAG1 and its mutant. Dual-luciferase reporter assay indicating the effect of miR-142-5p on WT JAG1 and its mutant in (B) AGS and (C) MKN-45 cells. (D and E) mRNA expression level of JAG1 in gastric cancer tissues and cells determined by reverse transcription-quantitative PCR. (F) Correlation analysis of miR-142-5p and JAG1 expression levels. (G) Protein level of JAG1 in AGS and MKN-45 cells transfected with miR-NC or miR-142-5p detected by western blot assay. "P<0.05. miR, microRNA; NC, negative control; NEAT1, nuclear-enriched abundant transcript 1; UTR, untranslated region; JAG1, jagged1; WT, wild-type; MUT, mutant.

MKN-45 cells after transfection with JAG1 overexpression plasmid compared with the pcDNA group (Fig. S2B). CCK-8 assay indicated that miR-142-5p overexpression suppressed the proliferation of gastric cancer cells, and overexpression of JAG1 abolished this effect (Fig. 6A and B). Similarly, the overexpression of JAG1 also reversed the miR-142-5p-mediated inhibitory effects on migration and invasion in gastric cancer cells (Fig. 6C and D). Western blot assay demonstrated that miR-142-5p decreased N-cadherin and Vimentin expression levels, but enhanced E-cadherin level in AGS and
MKN-45 cells compared with the miR-NC group, and when co-transfected with JAG1, this effect was reversed (Fig. 6E). Collectively, miR-142-5p inhibited the proliferation, migration and invasion of gastric cancer cells by targeting JAG1.

NEAT1 knockdown inhibits JAG1 expression in gastric cancer as a sponge of miR-142-5p. To elucidate the association between NEAT1 and JAG1 in gastric cancer cells, RT-qPCR and western blot assays were conducted, which indicated that the mRNA and protein expression levels of JAG1 were 
A

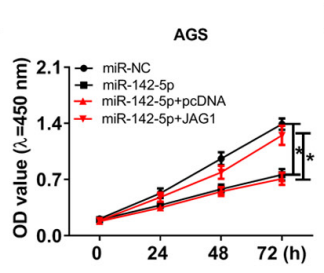

B

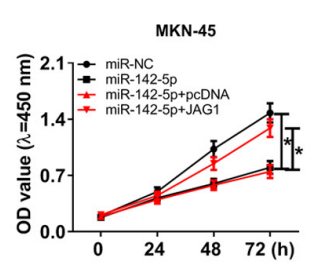

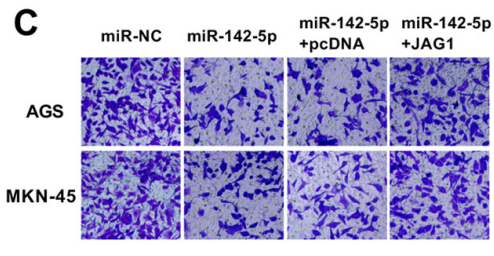

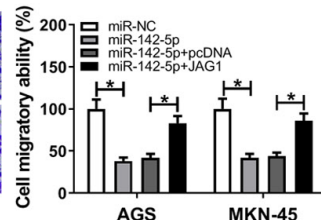

D

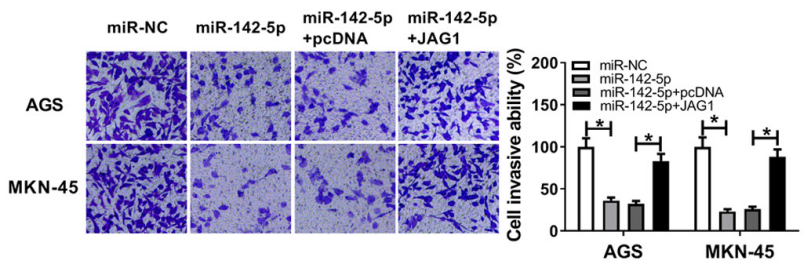

E

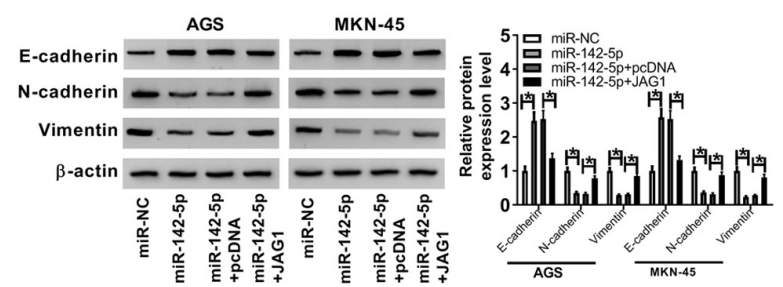

Figure 6. Effect of JAG1 overexpression on the miR-142-5p-mediated effects on proliferation, migration and invasion in gastric cancer cells. AGS and MKN-45 cells were transfected with miR-NC, miR-142-5p, miR-142-5p + pcDNA or miR-142-5p + JAG1. (A and B) Cell viability of AGS and MKN-45 cells at the indicated time points analyzed with Cell Counting Kit- 8 assay. (C and D) Migratory and invasive capabilities of AGS and MKN-45 cells examined by Transwell assay. (E) Protein expression level of E-cadherin, N-cadherin and Vimentin in transfected AGS and MKN-45 cells determined by western blot assay. ${ }^{*} \mathrm{P}<0.05$. miR, microRNA; NC, negative control; JAG1, jagged1.

A

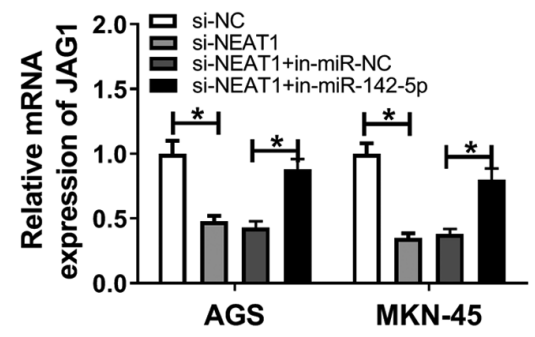

B

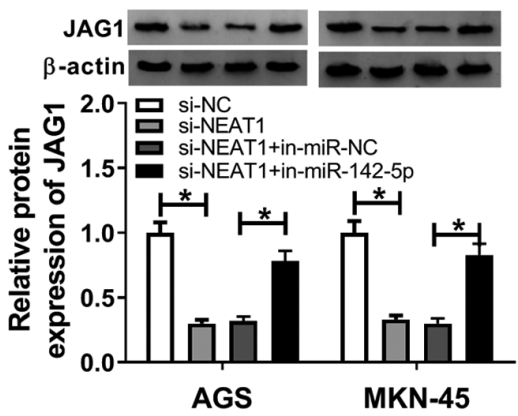

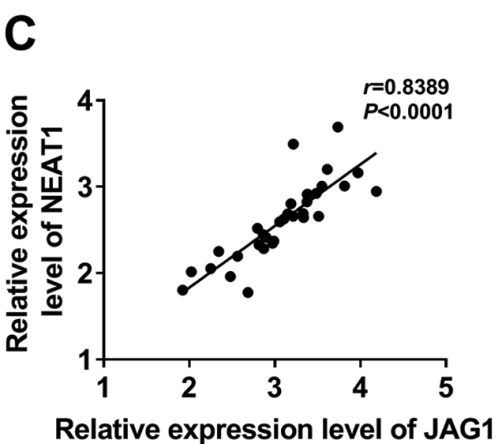

Figure 7. Effect of NEAT1 knockdown on JAG1 expression level in gastric cancer cells. (A and B) mRNA and protein expression levels of JAG1 in AGS and MKN-45 cells transfected with si-NC, si-NEAT1, si-NEAT1 + in-miR-NC or si-NEAT1 + in-miR-142-5p determined by reverse transcription-quantitative PCR and western blot assay, respectively. (C) Correlation analysis of JAG1 and NEAT1 expression levels in gastric cancer tissues. "P<0.05. NEAT1, nuclear-enriched abundant transcript 1; miR, microRNA; in, inhibitor of miR; si, small interfering; NC, negative control; JAG1, jagged1.

decreased in cells after NEAT1 knockdown, while transfection with miR-142-5p inhibitor effectively attenuated the NEAT1 silencing-induced reduced expression of JAG1 (Fig. 7A and B). A positive correlation between JAG1 and NEAT1 expression level in gastric cancer tissues was also observed (Fig. 7C). In conclusion, NEAT1 knockdown inhibited JAG1 expression by regulating miR-142-5p in vitro.

NEAT1 knockdown suppresses tumor growth and metastasis in vivo. A xenograft tumor mouse model was established to further investigate the functional effect of NEAT1 on tumor growth in vivo. As presented in Fig. 8A and B, NEAT1 knockdown resulted in an evident decrease in tumor volume and weight compared with the sh-NC group. NEAT1 expression level was lower in the tumor tissues of the sh-NEAT1 group compared with the sh-NC group (Fig. 8C). Moreover, RT-qPCR assay indicated that NEAT1 knockdown enhanced miR-142-5p expression level in tumor tissues (Fig. 8D). In addition, western blot analysis indicated that NEAT1 silencing significantly decreased the protein expression level of JAG1 (Fig. 8E). In addition, analysis of the protein levels of E-cadherin, $\mathrm{N}$-cadherin and Vimentin in xenograft tumor tissues revealed that E-cadherin expression level was increased, while N-cadherin and Vimentin levels were decreased in the sh-NEAT1 group compared with the sh-NC group (Fig. 8F). These results demonstrated that NEAT1 knockdown suppressed tumor grow th and metastasis in vivo by regulating miR-142-5p and JAG1 expression.

\section{Discussion}

In recent years, IncRNAs have attracted attention due to their potential as diagnostic and prognostic markers in numerous cancers (24). For example, Chen et al (25) reported that circPVT1 was a proliferative factor and independent prognostic biomarker for patients with gastric cancer. IncRNA maternally expressed 3 has been indicated to regulate gastric cancer progression via the p53 signaling pathway (26). In the present study, it was observed that high expression of NEAT1 was 

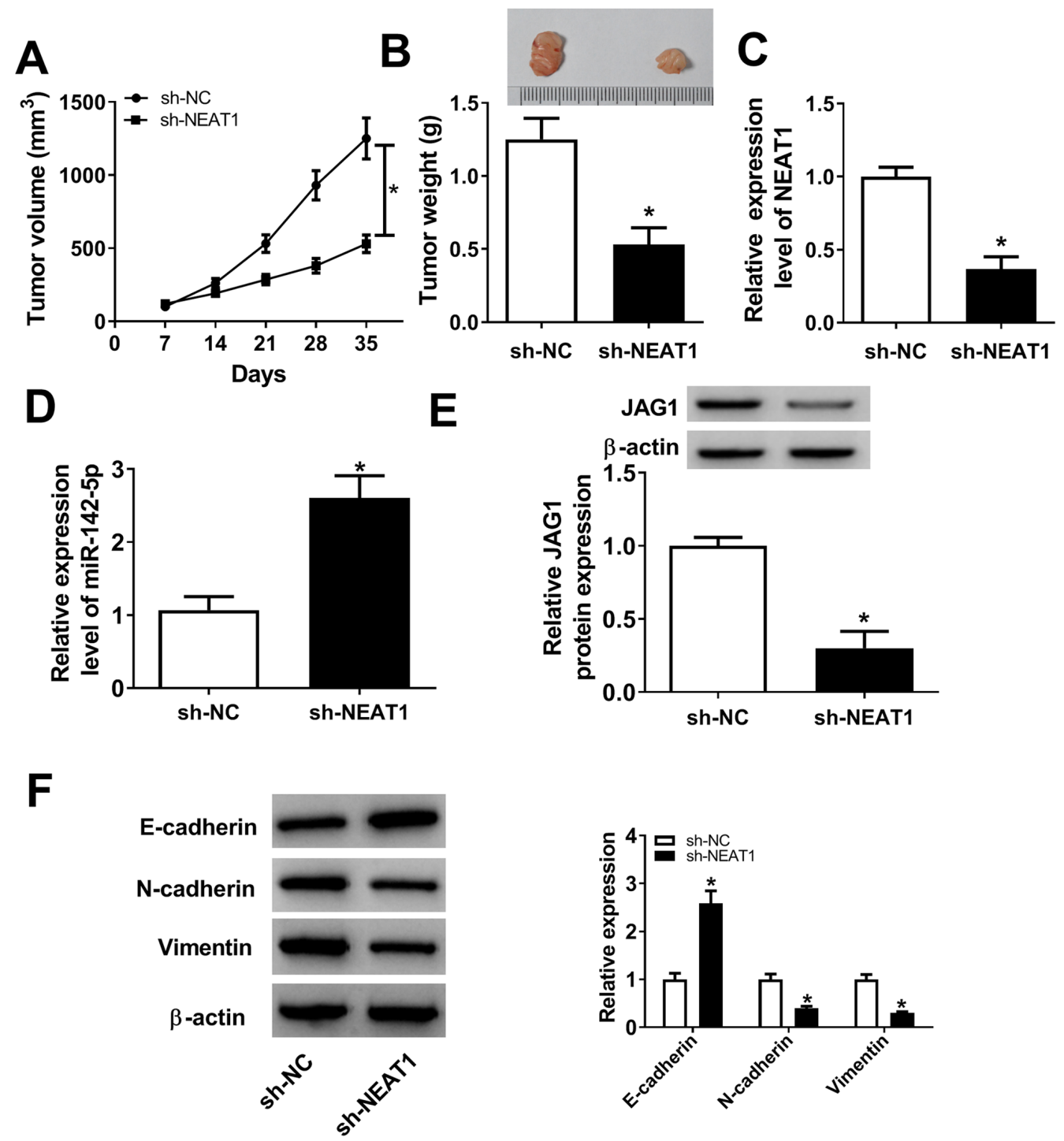

Figure 8. Effect of NEAT1 knockdown on tumor growth and JAG1 and miR-142-5p expression in tumor xenografts. (A and B) Growth curve of tumor volumes and weight. (C and D) Expression levels of NEAT1 and miR-142-5p in resected tumor tissues detected by reverse transcription-quantitative PCR. (E) Protein level of JAG1 in tumors determined by western blot assay. (F) Protein expression levels of E-cadherin, N-cadherin and Vimentin in tumors detected by western blot assay. "P<0.05. NEAT1, nuclear-enriched abundant transcript 1; miR, microRNA; sh, short hairpin; NC, negative control; JAG1, jagged1.

associated with poor survival of patients with gastric cancer. Functional experiments demonstrated that NEAT1 silencing or miR-142-5p overexpression inhibited cell migration and invasion, but these effects could be reversed by miR-142-5p inhibitor or JAG1 overexpression, respectively. Additionally, the current study used both AGS and MKN-45 gastric cancer cell lines, and the results were similar in the two cell lines, suggesting the relevance of the present results in gastric cancer. However, certain results differed between the AGS and MKN-45 gastric cancer cell lines. For example, the levels of NEAT1 and JAG1 were lower, but the level of miR-142-5p was higher in AGS cells compared with that in MKN-45 cells, which may be attributed to the different malignant potential of the two cell lines.

A previous study demonstrated that patients with gastric cancer and high level of NEAT1 exhibited poor survival (27). Therefore, NEAT1 may be used as a therapeutic target and prognostic biomarker for gastric cancer. A negative association between the high level of NEAT1 and the five-year survival rate of patients with gastric cancer was also observed. Fu et al (27) demonstrated that NEAT1 was upregulated in gastric cancer tissues and cell lines, and knockdown of NEAT1 suppressed gastric cancer cell migration and invasion in vitro. Another study also revealed that NEAT1 knockdown inhibited the progression of gastric cancer (28). Consistent with these studies, the present study also demonstrated that knockdown of NEAT1 suppressed the proliferation, migration and invasion of gastric cancer cells in vitro, while these inhibitory effects could be abrogated by miR-142-5p inhibitor.

A previous study has indicated that miRNAs functioned as biomarkers, which may enhance the sensitivity and specificity of gastric cancer diagnostic and prognostic tests (29). Zhang et al (30) revealed that a low level of hsa-miR-142-5p in gastric cancer was associated with high frequency recurrence and poor survival. In the present study, miR-142-5p was observed to be decreased in gastric cancer cells and identified as a target of NEAT1. Further functional experiments revealed that miR-142-5p silencing restored 
the NEAT1 knockdown-induced effects on proliferation, migration and invasion in gastric cancer cells. Shen et al (31) reported that miR-142-3p was markedly decreased in colon cancer cells and inhibited gene expression by binding to the 3'-UTR of the CD133. In the current study, mechanistic experiments revealed that miR-142-5p could bind to the 3'-UTR of JAG1.

JAG1 is one of the most important ligands of the Notch signaling pathway (19). Increasing evidence has suggested that dysregulation of JAG1 was involved in the development of malignant tumors (32). Jiang et al (15) also revealed the important role of JAG1 in gastric cancer, demonstrating that the upregulation of miR-124 inhibited gastric cancer cell growth, migration and invasion by regulating JAG1 expression. In the present study, the expression level of miR-142-5p was inversely correlated with JAG1 expression level in patients with gastric cancer. Overexpression of miR-142-5p impeded gastric cancer cell growth, migration and invasion, which could be reversed by overexpression of JAG1. Moreover, it was revealed that upregulation of miR-142-5p could decrease the expression level of JAG1 in gastric cancer cells, suggesting that miR-142-5p may inhibit gastric cancer development via directly targeting JAG1.

Finally, the biological functions of NEAT1 were further investigated on tumor growth in vivo, and the results indicated that NEAT1 silencing suppressed tumor growth and metastatic potential in vivo via regulating the miR-142-5p/JAG1 axis. However, there are also certain limitations to the present study. The number of clinical samples was low, which may lead to an inaccurate result. Moreover, the effect of the NEAT1/miR-142-5p/JAG1 axis on the Notch signaling pathway has not been explored. In addition, whether the NEAT1/miR-142-5p/JAG1 axis could regulate drug resistance in gastric cancer also requires further elucidation.

To conclude, increased NEAT1 and JAG1 expression levels, as well as decreased miR-142-5p level were observed in gastric cancer tissues compared with neighboring healthy tissues. Functionally, NEAT1 contributed to gastric cancer progression via targeting miR-142-5p to regulate JAG1 expression in vitro and in vivo, which indicated that NEAT1 and miR-142-5p may serve as promising therapeutic targets for the treatment of gastric cancer.

\section{Acknowledgements}

Not applicable.

\section{Funding}

No funding was received.

\section{Availability of data and materials}

The datasets used and/or analyzed during the current study are available from the corresponding author on reasonable request.

\section{Authors' contributions}

YZ: Conceptualization. LC and QW: Acquisition of data. YZ and FZ: Analysis of data and data curation. YZ and FZ:
Confirming the authenticity of the raw data. All authors: Read and approved the final manuscript.

\section{Ethics approval and consent to participate}

The current study was approved by the Ethics Committee of Yantaishan Hospital (Yantai, China), and a written informed consent form was obtained from each patient. The Institutional Animal Care and Use Committee of Yantaishan Hospital (Yantai, China) authorized all animal experiments.

\section{Patient consent for publication}

Not applicable.

\section{Competing interests}

The authors declare that they have no competing interests.

\section{References}

1. Smyth EC, Nilsson M, Grabsch HI, van Grieken NC and Lordick F: Gastric cancer. Lancet 396: 635-648, 2020.

2. Bray F, Ferlay J, Soerjomataram I, Siegel RL, Torre LA and Jemal A: Global cancer statistics 2018: GLOBOCAN estimates of incidence and mortality worldwide for 36 cancers in 185 countries. CA Cancer J Clin 68: 394-424, 2018.

3. Du X, Cheng Z, Wang YH, Guo ZH, Zhang SQ, Hu JK and Zhou ZG: Role of Notch signaling pathway in gastric cancer: A meta-analysis of the literature. World J Gastroenterol 20: 9191-9199, 2014.

4. Dey BK, Mueller AC and Dutta A: Long non-coding RNAs as emerging regulators of differentiation, development, and disease. Transcription 5: e944014, 2014.

5. Shi X, Sun M, Liu H, Yao Y and Song Y: Long non-coding RNAs: A new frontier in the study of human diseases. Cancer Lett 339: 159-166, 2013.

6. Pan LJ, Zhong TF, Tang RX, Li P, Dang YW, Huang SN and Chen G: Upregulation and clinicopathological significance of long non-coding NEAT1 RNA in NSCLC tissues. Asian Pac J Cancer Prev 16: 2851-2855, 2015.

7. Li Y, Chen D, Gao X, Li X and Shi G: LncRNA NEAT1 regulates cell viability and invasion in esophageal squamous cell carcinoma through the miR-129/CTBP2 axis. Dis Markers 2017: $5314649,2017$.

8. Wu Y, Yang L, Zhao J, Li C, Nie J, Liu F, Zhuo C, Zheng Y, Li B, Wang $\mathrm{Z}$ and $\mathrm{Xu} \mathrm{Y}$ : Nuclear-enriched abundant transcript 1 as a diagnostic and prognostic biomarker in colorectal cancer. Mol Cancer 14: 191, 2015.

9. Mang Y, Li L, Ran J, Zhang S, Liu J, Li L, Chen Y, Liu J, Gao Y and Ren G: Long noncoding RNA NEAT1 promotes cell proliferation and invasion by regulating hnRNP A2 expression in hepatocellular carcinoma cells. Onco Targets Ther 10: 1003-1016, 2017.

10. Zhen L, Yun-Hui L, Hong-Yu D, Jun M and Yi-Long Y: Long noncoding RNA NEAT1 promotes glioma pathogenesis by regulating miR-449b-5p/c-Met axis. Tumour Biol 37: 673-683, 2016.

11. Gong W, Zheng J, Liu X, Ma J, Liu Y and Xue Y: Knockdown of NEAT1 restrained the malignant progression of glioma stem cells by activating microRNA let-7e. Oncotarget 7: 62208-62223, 2016.

12. Chen X, Kong J, Ma Z, Gao S and Feng X: Up regulation of the long non-coding RNA NEAT1 promotes esophageal squamous cell carcinoma cell progression and correlates with poor prognosis. Am J Cancer Res 5: 2808-2815, 2015.

13. Tafrihi M and Hasheminasab E: miRNAs: Biology, biogenesis, their web-based tools, and databases. Microrna 8: 4-27, 2019.

14. Liu Y, Xing R, Zhang X, Dong W, Zhang J, Yan Z, Li W, Cui J and $\mathrm{Lu} \mathrm{Y}$ : miR-375 targets the p53 gene to regulate cellular response to ionizing radiation and etoposide in gastric cancer cells. DNA Repair (Amst) 12: 741-750, 2013.

15. Jiang L, Lin T, Xu C, Hu S, Pan Y and Jin R: miR-124 interacts with the Notch1 signalling pathway and has therapeutic potential against gastric cancer. J Cell Mol Med 20: 313-322, 2016. 
16. Xie L, Zhang Z, Tan Z, He R, Zeng X, Xie Y, Li S, Tang G, Tang $\mathrm{H}$ and He $\mathrm{X}$ : MicroRNA-124 inhibits proliferation and induces apoptosis by directly repressing EZH2 in gastric cancer. Mol Cell Biochem 392: 153-159, 2014.

17. Hu CB, Li QL, Hu JF, Zhang Q, Xie JP and Deng L: miR-124 inhibits growth and invasion of gastric cancer by targeting ROCK1. Asian Pac J Cancer Prev 15: 6543-6546, 2014.

18. Ma Z, Liu T, Huang W, Liu H, Zhang HM, Li Q, Chen Z and Guo AY: MicroRNA regulatory pathway analysis identifies miR-142-5p as a negative regulator of TGF- $\beta$ pathway via targeting SMAD3. Oncotarget 7: 71504-71513, 2016.

19. Grochowski CM, Loomes KM and Spinner NB: Jagged1 (JAG1): Structure, expression, and disease associations. Gene 576 381-384, 2016.

20. Jia CM, Tian YY, Quan LN, Jiang L and Liu AC: miR-26b-5p suppresses proliferation and promotes apoptosis in multiple myeloma cells by targeting JAG1. Pathol Res Pract 214: 1388-1394, 2018

21. Xiao HJ, Ji Q, Yang L, Li RT, Zhang C and Hou JM: In vivo and in vitro effects of microRNA-124 on human gastric cancer by targeting JAG1 through the Notch signaling pathway. J Cell Biochem 119: 2520-2534, 2018.

22. Livak KJ and Schmittgen TD: Analysis of relative gene expression data using real-time quantitative PCR and the 2(-Delta Delta $\mathrm{C}(\mathrm{T})$ ) method. Methods 25: 402-408, 2001.

23. Ministry of Science and Technology of the People's Republic of China. Guide for the care and use of laboratory animals. Ministry of Science and Technology of the People's Republic of China, Beijing, People's Republic of China (In Chinese).

24. Chandra Gupta S and Nandan Tripathi Y: Potential of long non-coding RNAs in cancer patients: From biomarkers to therapeutic targets. Int J Cancer 140: 1955-1967, 2017.
25. Chen J, Li Y, Zheng Q, Bao C, He J, Chen B, Lyu D, Zheng B, $\mathrm{Xu} Y$, Long Z, et al: Circular RNA profile identifies circPVT1 as a proliferative factor and prognostic marker in gastric cancer. Cancer Lett 388: 208-219, 2017.

26. Wei GH and Wang X: lncRNA MEG3 inhibit proliferation and metastasis of gastric cancer via p53 signaling pathway. Eur Rev Med Pharmacol Sci 21: 3850-3856, 2017.

27. Fu JW, Kong Y and Sun X: Long noncoding RNA NEAT1 is an unfavorable prognostic factor and regulates migration and invasion in gastric cancer. J Cancer Res Clin Oncol 142: 1571-1579, 2016.

28. Zhang J, Zhao B, Chen X, Wang Z, Xu H and Huang B: Silence of long noncoding RNA NEAT1 inhibits malignant biological behaviors and chemotherapy resistance in gastric cancer. Pathol Oncol Res 24: 109-113, 2018

29. Liu HS and Xiao HS: MicroRNAs as potential biomarkers for gastric cancer. World J Gastroenterol 20: 12007-12017, 2014.

30. Zhang X, Yan Z, Zhang J, Gong L, Li W, Cui J, Liu Y, Gao Z, $\mathrm{Li}$ J, Shen L and Lu Y: Combination of hsa-miR-375 and hsa-miR$142-5 p$ as a predictor for recurrence risk in gastric cancer patients following surgical resection. Ann Oncol 22: 2257-2266, 2011.

31. Shen WW, Zeng Z, Zhu WX and Fu GH: miR-142-3p functions as a tumor suppressor by targeting CD133, ABCG2, and Lgr5 in colon cancer cells. J Mol Med (Berl) 91: 989-1000, 2013.

32. Li D, Masiero M, Banham AH and Harris AL: The notch ligand JAGGED1 as a target for anti-tumor therapy. Front Oncol 4: 254, 2014. International (CC BY-NC-ND 4.0) License. 